\title{
The realization of the agricultural expert system in embedded platform
}

\author{
Yunfei Zhang, Zhixing Shi, Zheng Xu \\ College of Information Science and Technology, Agricultural University of Hebei, Baoding,Hebei 071001
}

\begin{abstract}
In order to improve the utilization of the Agricultural Expert System so that it can be taken to the fields directly to guide agricultural production. The development and implementation of the portable Agriculture Expert System are studied in this paper,especially,the image acquisition is proposed as the information input for the human-computer interaction, and process of the cameras capturing the images is described in detail,so Agricultural Expert System ,operated more easier,can guaid peasant production more directly.

Index Terms - Expert System, Portable,Capturing images.
\end{abstract}

\section{Introduction}

The expert system is a branch of artificial intelligence technology, the application of expert systems is one of the focus fields of agriculture research. Early in 1970s, expert system used in the field of agriculture already existed[1]. After more than 40 years of development, agricultural expert system has been applied to all aspects of agriculture, such as crop cultivation and management, Horticultural management, animal husbandry, aquaculture, resource protection, Farming and farm management and decision-making and so on. However, the development of the agricultural expert systems are based on the PC platform, it is inconvenient to be brought to the fields to direct guidance of agricultural production, Secondly, the national conditions of China and developed countries are very different. Therefore, these countries place more emphasis on how to improve the resource utilization, agricultural economic efficiency and environmental protection to achieve sustained development aspects of technology research and application, the levels of agricultural information and labor productivity is also higher. In China, the vast rural areas is a serious lack of high-level agricultural experts in the field of science and technology personnel, means of communication backward, low education level of farmers, and our small farmers farmland area, the basic conditions of the various plots, farming around the technical differences, thus leading to China's new agricultural technology, new achievements promote slow[2]. In addition, the low level of China's rural culture, farmers there are difficulties in the use of expert systems for information input also.These have led to the agricultural expert system application is limited.

Therefore, portable agricultural expert system has become a hot topic[3]. Based on the strong function, low cost, small size, low power consumption for embedded systems[4], the development of a portable agricultural expert system, and propose a new information input, image acquisition information input. This will allow the agricultural expert system can direct guidance of first-line farmers and more convenient to use.

\section{Expert system analysis}

Portable agricultural expert system will be used more direct and more widely. However, in the expert system development process, we also need to solve some problems. The expert system to run the environment is the primary problem, choose a powerful, reliable software system is the basis of assurance expert system running smoothly. The expert system diagnostic accuracy of the critical process that expert system's knowledge acquisition is still in the construction process of the expert system system a "bottleneck"[5]. Expert system contains a knowledge base, database and other large amounts of data, a handheld device have sufficient storage capacity is the operation of the key by the expert system. Currently, human-computer interaction for most of the expert system to select the input or text input form, which is obviously bring inconvenience to the use of the low education level of farmers' friends. In order to ensure the widespread use of the agricultural expert system to solve these problems in a priority.

\section{The hardware platform of the system}

The system hardware platform such as the one shown in the figure below.

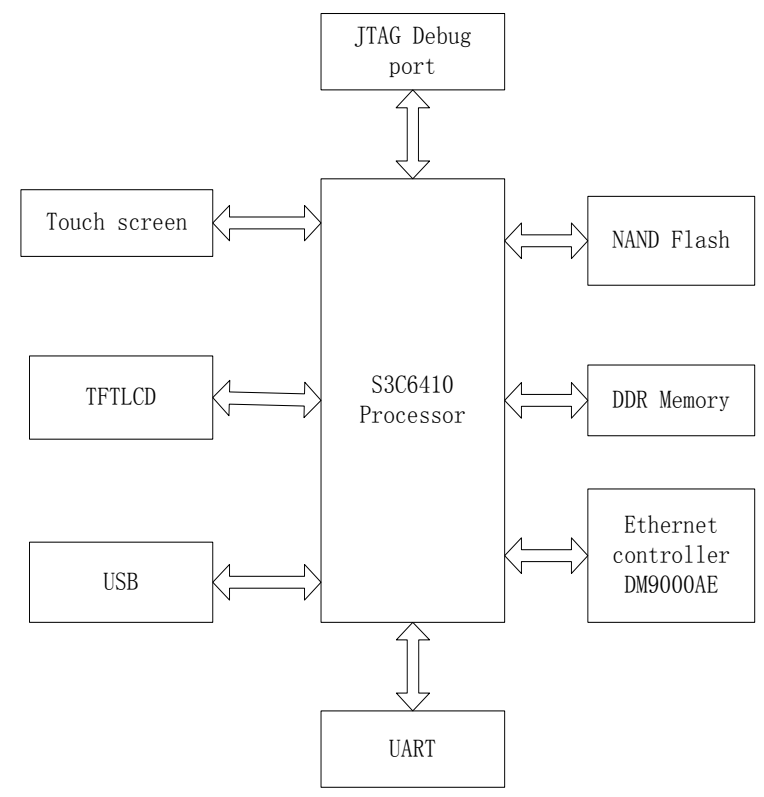

Fig. 1 Hardware system block diagram 
The expert system uses the the Feiling company S3C6410 development board. First, S3C6410 (ARM11) processor support superb performance for portable applications. It has an independent load-store and eight arithmetic pipeline, and compared to ARM9, the performance increased by approximately $40 \%$. In addition, it is a high-performance, low-power 'quasi-64' microprocessor, allowing processor for two instructions and perform multiple data reading and writing instruction at the same time in a single clock cycle. It greatly improving the speed of code to run. Second, the development board also provides $2 \mathrm{G}$ bytes of NAND Flash memory, with 256M DDR memory, and to ensure the security and efficient storage of large amounts of data. In addition, it also supports TFT LCD and touch screen, as well as to expand outside the USB host port controller, etc., and they constitute an embedded multimedia development platform, to meet the complex computing and graphics display needs.

\section{The design of Agricultural expert system}

\section{A. The frame of Agricultural expert system}

Agricultural Expert System is composed mainly by database, knowledge base, explain the mechanism, reasoning mechanism, and human-computer interaction interface [6-7]. The structural framework shown in Figure 2.

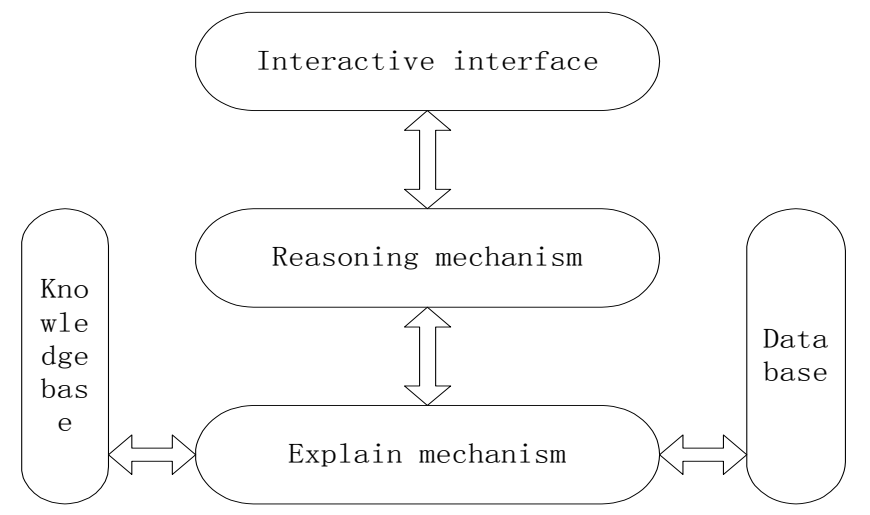

Fig. 2 Basic block diagram of the expert system

\section{B. The knowledge base based on data mining technology}

In this paper, the design of jujube pest diagnosis expert system using SQL Server to build expert knowledge base, and potential law model from massive data mining using data mining techniques. Data mining technology is a process of decision-support which to find mode in some fact or observation data collection. Expert system model based on data mining requires that a large number of data ran by the system. This is the database basis of the system running[8]. System knowledge base is divided into two types: one is the historical database, that library before jujube pest; another rule base knowledge, there is recorded with a variety of constraints and inference rules, is an understanding of the conditions and judgments of expert optimization on the unit. The manmachine dialogue interface given multiple input information. The expert system began reasoning by the signal, using logical reasoning deduction, under limited conditions, filter through large amounts of data from which to explore the optimal unit commitment. Among them, the associated working methods of data mining technology will be used in the realization of logical reasoning.

\section{The inference engine based on forward reasoning}

Expert system has more reasoning and classification. By reasoning conclusions and the target closeness can be divided into monotonic reasoning and nonmonotonic reasoning; By reasoning with knowledge is determined, can be divided into deterministic reasoning and uncertainty reasoning; By direction of reasoning can be divided into forward reasoning and backward reasoning. Jujube pest diagnosis expert system designed in this paper, adopted the forward reasoning[9].

Forward reasoning, also known as the the antecedent reasoning or data-driven, the basic principle: start with the fact of problem, forward using rules, when the condition part of the rules match with the facts, put the rule into the candidate as an available rule, and then through conflict resolution, select a rule in the candidate queue for further reasoning as start rule. Repeat the above process until no available rules or to obtain the desired results so far. In this paper, the design of the reasoning process of the expert system is shown in Figure 3.

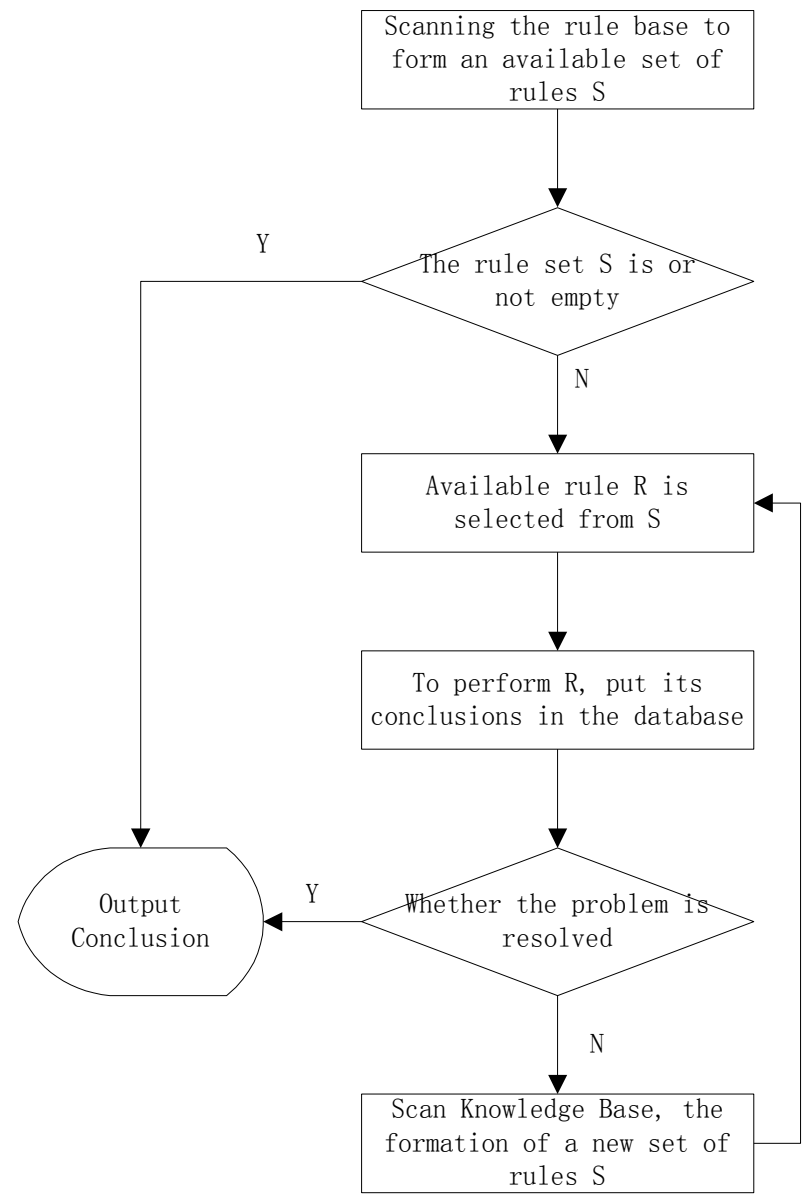

Fig. 3 Forward reasoning processes of expert systems

\section{Information Input of the Expert System}

1) Select the input

The expert system is the diagnostic expert system, and select the input is the most basic way to input information. 
That is, when the farmers enter into the expert system, they can select the entry according to the jujube diseased parts (such as leaves). After entering the system, it will prompt to select the input incidence period (such as the early), In this way, the layers of selection information input to determine the dates of the disease, and to give appropriate explanations and prevention information. Thereby the expert system is able to diagnose diseases of the dates.

\section{2) Image Acquisition Input}

In addition to select the input, this paper proposes a new information input mode, as an information input by the image acquisition. Currently, many expert system enter information by entering text as the main way, however, due to the low level of China's rural culture, there are still some inconveniences for the farmers friends. If farmers use portable expert system such as using a camera to take pictures of plant disease site, then the expert system can feedback the corresponding disease explanation and prevention information, this will make the use of the expert system becomes more convenient.

The expert system increases a camera on the handheld device to achieve this functionality. The camera is a USB device, and the USB device support in the Linux system is shown in Figure 4. USB Core contains all USB devices and some general operation of the host controller, and provides an API interface for the device and the host controller. USB device drivers interact with the device through providing a standard interface by the USB Core. It uses channels by pipeline to communicate with device[10]. In Linux system, the general file operations are done through the system calls, These system calls are implemented by data copying between kernel mode and user mode. For image data, the copy will definitely increase the time overhead, therefore, solve the problem by the memory-mapped . First, apply for a sufficiently large kernel mode as the image data buffer, and the USB request block data is temporarily stored, then it be mapped to user space of states. So that the image processing program can use mmap () function directly read and write kernel mode frame buffer, and reduce the additional spending. Such the system capture the image through the camera, and then transport it to the embedded device processor through the USB bus for processing, and compresses image, saved as JPEG files. Then extract useful information through the latter part of the image processing and analysis, and match the information in the knowledge base of expert system, and finally feedback to the user Jujube disease information and prevention methods to achieve the purpose of diagnosis.

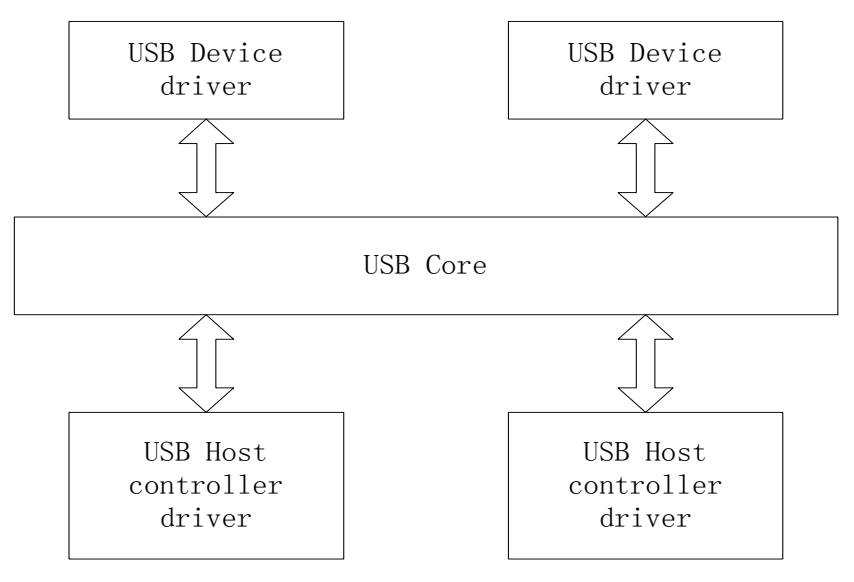

Fig.4 The USB system software structure of Linux

\section{Conclusion}

This paper designed and implemented expert system based on embedded devices, and proposes the use of image capture as an information input, Solve the basic work and camera capture images of the expert system running, then laid the foundation for the development of portable expert system. However, due to my limited research capacity, Post-processing image and with the combination of expert system remains to be to continue to improve.

\section{References}

[1] Lin Shi, Diyi Chen, Xiaoyi Ma, Overview and prospects of the application of expert systems in agriculture, Journal of Agricultural Mechanization Research , 2011.

[2] Jianquan Ouyang, The Design and Implement of PDA-Oriented Expert System in Agriculture, Computer Engineering and Applications, Feb 2002.

[3] Liping Chen, Donghui Wang, Chunjiang Zhao, Research and Development of a Development Platform on Handheld Personal Computer for Agriculture Expert System, Transactions of the Chinese Society of Agricultural Engineering, 2002.

[4] Baowei Cheng, Overview of Embedded Systems and their Development and Application, Journal of Yangtze University, 2004.

[5] Yongqing Wang, Principles of Artificial Intelligence and Methods, Xi'an Jiaotong University Press, 2003.

[6] Zixing Cai, Guangyou Xu, Artificial Intelligence and Applications, Tsinghua University Press, 2003.

[7] Jianlong Li, Information Agroecology, Chemical Industry Press, 2004.

[8] Jiawei Han, Micheline Kamber, Data Mining, Machinery Industry Press, 2001.

[9] Yanan Wang, Research and Application of Expert System Inference Mechanism, Wuhan University of Technology, 2006.

[10] Zhaoqing Li, Feng Liao, Jiancun Liu, USB Interface Technology, National Ddfence Industry Press, 2004. 